\title{
Estudio comparativo entre los ciclos 38 y 40 del modelo Harmonie-Arome para la zona de Canarias
}

https://doi.org/10.31978/639-19-010-0.575

\author{
Irene Recuerda Gavilán' (irecuerdag@aemet.es) \\ David Suárez Molina1 (dsuarezm@aemet.es)
}

${ }^{1}$ AEMET / Delegación Territorial en Canarias

\begin{abstract}
RESUMEN
En las islas Canarias, especialmente debido a su compleja orografía, se hace necesario el uso del modelo de predicción numérica (NWP) determinista no hidrostático de alta resolución de AEMET, Harmonie-Arome, para la emisión de avisos por fenómenos meteorológicos adversos (FMA). Por tanto, el objetivo de este trabajo es presentar los resultados de una evaluación comparativa de la calidad de predicciones de alta resolución proporcionadas por los ciclos 40 (operativo) y 38 (previo) de este modelo en la zona de Canarias. Para ello, se han elegido tres casos de estudio del primer trimestre de 2018 con FMA, en los que se emitieron avisos amarillos/naranjas con diferentes configuraciones de viento. Los campos de viento a 10 metros, rachas de viento y precipitación se han analizado y comparado con las respectivas observaciones registradas. Por último, se evaluarán también diagramas de dispersión. Así, los estudios regionalizados de diferentes modelos en situaciones de FMA permiten mitigar la controversia a la que deben enfrentarse los predictores operativos a la hora de tomar decisiones para la emisión de avisos.
\end{abstract}

PALABRAS CLAVE: predicción; casos de estudio; fenómenos meteorológicos adversos; avisos; Harmonie-Arome; Canarias.

\section{INTRODUCCIÓN}

Una de las actividades fundamentales de los Grupos de Predicción y Vigilancia de AEMET es la emisión de avisos por fenómenos meteorológicos adversos (FMA). Para ello, los predictores operativos cuentan con los modelos de predicción numérica (NWP) como herramienta fundamental.

Las islas Canarias se caracterizan por una compleja orografía de origen volcánico, con montañas muy elevadas en comparación con la extensión superficial del archipiélago. Todas las islas, con excepción de Lanzarote y Fuerteventura, poseen cumbres centrales dominantes desde las que se descuelgan laderas de gran pendiente y profundos barrancos hasta llegar al mar. Esta disposición del relieve, junto con las características que supone el ser un territorio insular situado en la zona subtropical del océano Atlántico, hace necesario el uso de un modelo numérico de alta resolución, que refleje, en la medida de lo posible, estas particularidades.

En AEMET, el modelo determinista no hidrostático de alta resolución operativo es Harmonie-Arome. Araíz de la implantación del nuevo ciclo 40 (Har40), operativo desde el 1 de junio de 2017, se han observado notables diferencias respecto al anterior ciclo 38 (Har38).

El objetivo de este trabajo es realizar una evaluación comparativa con respecto a la calidad de las predicciones de alta resolución proporcionadas por los ciclos 38 y 40 del modelo no hidrostático Harmonie-Arome a 
2,5 km de resolución en la zona de Canarias. Se han elegido tres casos de estudio pertenecientes a los primeros meses de 2018 con FMA en los que se emitieron avisos amarillos/naranja y se analizarán las rachas de viento y precipitación pronosticadas comparando con las respectivas observaciones.

\subsection{Modelo Harmonie-Arome: ciclos 38 y 40}

Harmonie-Arome es una configuración dentro del sistema compartido de colaboración entre los consorcios ALADIN y HIRLAM (BENGTSSON et al., 2017). El modelo Harmonie-Arome tiene una resolución horizontal de 2,5 km y 65 niveles verticales. Se caracteriza por ser un modelo no hidrostático, que resuelve explícitamente la convección profunda, con una asimilación de datos importante en el sistema. Además, se presenta con una dinámica espectral muy eficiente para usos operativos.
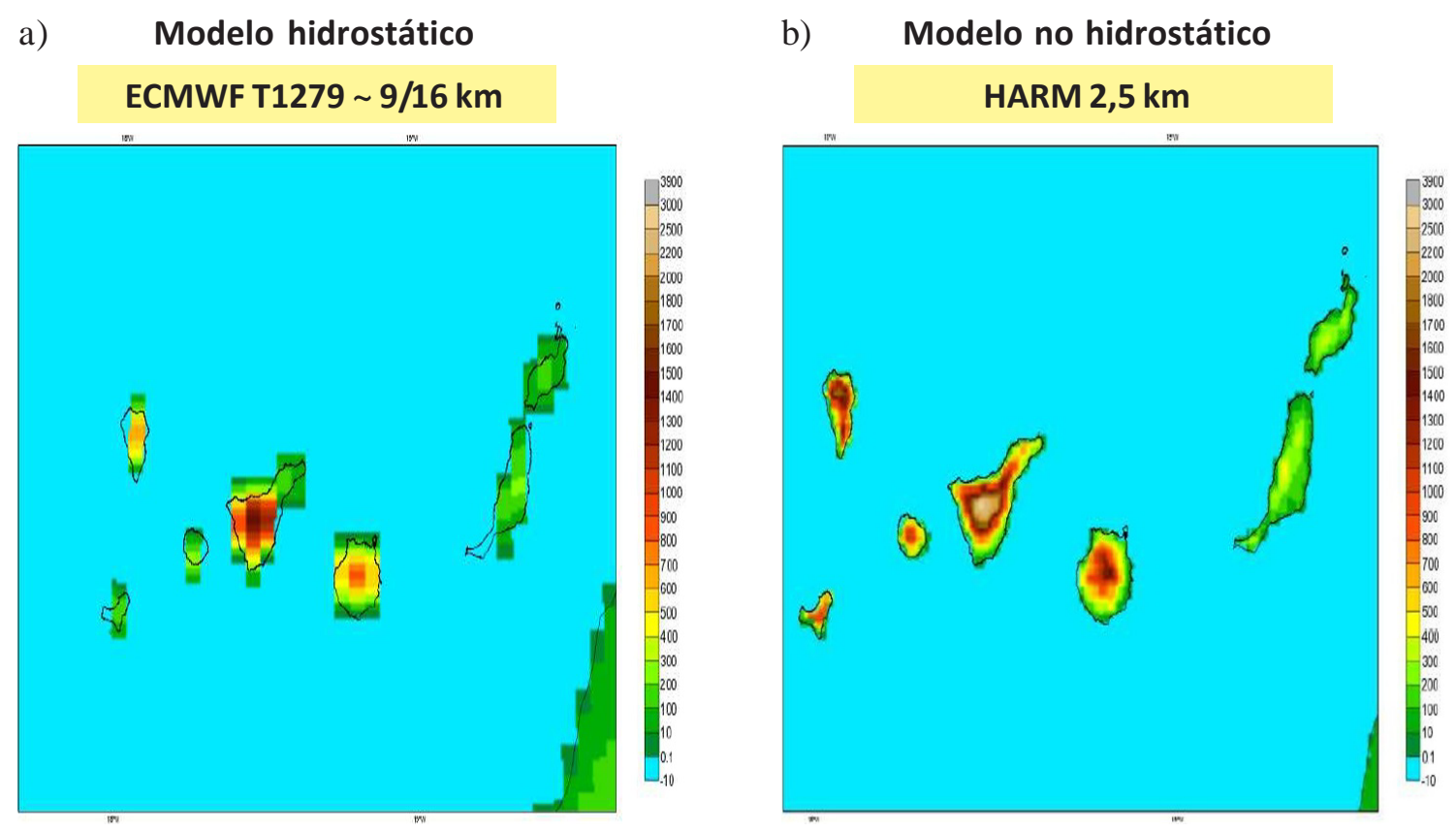

Figura 1. a) Orografía del modelo hidrostático ECMWF y

b) del modelo no hidrostático Harmonie-Arome para las islas Canarias.

El ciclo 38h1.1 (Har38) ha sido la configuración operativa hasta junio 2017. Este ciclo dispone de 4 pasadas al día cada 6 horas, con un alcance de predicción H+48 y ciclos de asimilación de datos cada 6 horas. En Canarias solo dispone de análisis en superficie, inicializándose en altura con la predicción $\mathrm{H}+6$ del modelo del Centro Europeo (ALADIN-HIRLAM, 2012).

A partir de entonces, entró en vigor el ciclo 40h1.1 (Har40) (ALADIN-HIRLAM, 2014), que dispone de 8 pasadas al día cada 3 horas y alcance de predicción $\mathrm{H}+48$, con salidas cada 15 minutos para las variables de superficie seleccionadas. Otros cambios significativos fueron:

- Cambios significativos en el esquema de turbulencia y en la microfísica.

- Asimilación de datos cada 3 horas: más observaciones y con mayor precisión temporal; también en altura, incluyendo observaciones convencionales, sondeos, aviones y ATOVS (satélites).

\subsection{Verificación y diagramas de dispersión}

Realizar una predicción se asemeja a hacer un experimento, es decir, dado un conjunto de condiciones, se plantean hipótesis acerca de si un hecho futuro ocurrirá o no. El experimento no se considerará completo 
hasta que se determina qué ocurrió finalmente. De la misma forma ocurre con una predicción del tiempo, no se debe considerar completa hasta que se descubre lo que sucedió en realidad. Por tanto, existen tres razones para hacer verificaciones (WWRP/WGNE JoInt Working Group on ForeCAST Verification ResEarch, 2017):

- Controlar la calidad de la predicción: cómo de precisas fueron las predicciones y si van mejorando con el tiempo.

- Mejorar la calidad de la predicción: para lo que es necesario estudiar los aciertos y fallos en las predicciones realizadas.

- Comparar la calidad con diferentes sistemas de predicción: hasta qué punto un sistema de predicción proporciona mejores predicciones que otro, y en qué sentido este sistema es mejor.

Los métodos visuales son unos de los métodos más antiguos: mirar la predicción y la observación por separado y utilizar el juicio humano para discernir los errores y diferencias. Para ello se suelen usar mapas y series temporales. Aunque este es un método no cuantitativo y está supeditado a una interpretación individual o subjetiva, incluso cuando se hace estadística, a veces es buena idea echar un vistazo a los datos.

Una forma de visualización de todos los datos del conjunto estadístico son los diagramas de dispersión (figura 2), que muestran el grado de correlación entre las dos variables del conjunto. En el caso de la citada figura, para los tres primeros meses de 2018.

A priori, se puede observar cómo, para las rachas, la distribución de Har38 se acerca más a la diagonal, que sería la de un pronóstico ideal, mientras que Har40 parece hacer una sobrestimación general. Respecto al diagrama de dispersión de la precipitación, hay menos casos para hacer la comparación, pero parece que, en este caso, Har38 es el que realiza una sobrestimación de la intensidad de precipitación, mientras que los datos más centrados en la diagonal los proporciona Har40.
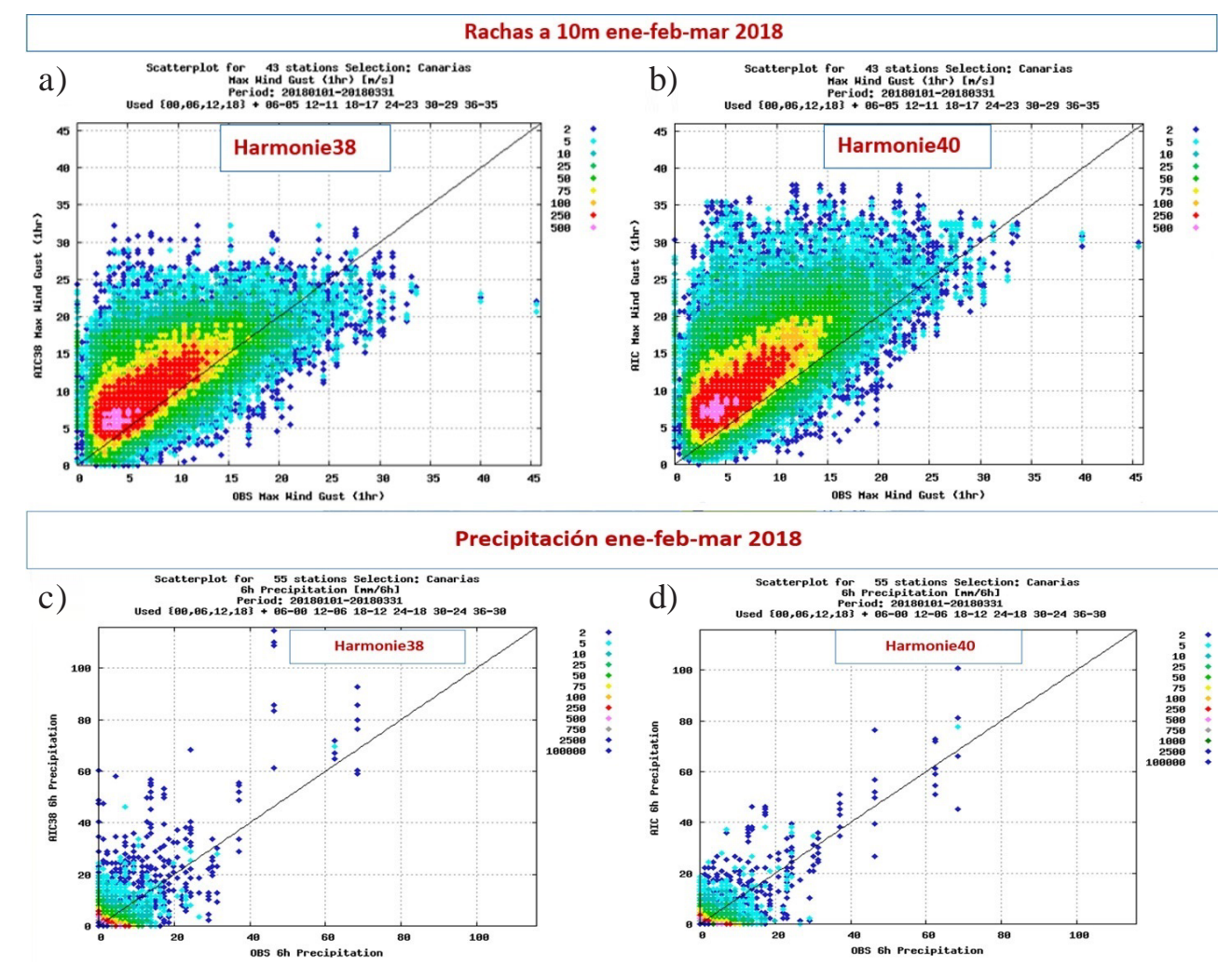

Figura 2. Diagramas de dispersión para a) rachas máximas de viento a $10 \mathrm{~m}$ en $1 \mathrm{~h}(\mathrm{~m} / \mathrm{s})$ observadas frente a las pronosticadas por Harmonie-Arome ciclo 38 y b) ciclo 40; c) precipitación acumulada en $6 \mathrm{~h}$ (mm/6 h) observadas frente a las pronosticadas por Harmonie-Arome ciclo $38 \mathrm{y}$ d) para ciclo 40, para el periodo enero, febrero y marzo de 2018. (Fuente: Área de Modelización, AEMET). 


\section{CASOS DE ESTUDIO}

Veamos, entonces, algunos casos de estudio seleccionados en este periodo del primer trimestre de 2018. Se han elegido tres casos, pertenecientes a enero y febrero de 2018, con FMA en los que se emitieron avisos amarillos/naranjas: una situación de alisio o viento del nordeste intenso (viento de tiempo normal en Canarias), una situación con vientos del suroeste (inusual) y, por último, una situación de vientos del norte-noroeste (relativamente frecuentes). Los datos reales usados para la comparación son los medidos con las estaciones automáticas de la red de AEMET.

\subsection{Día 25/02/2018. Viento del SW (aviso naranja)}

Durante la semana previa, los modelos predecían la entrada de una borrasca atlántica. El anticiclón de las Azores que sirvió de bloqueo durante los meses anteriores, se debilitó y se desplazó hacia el interior del continente europeo, permitiendo la entrada de borrascas atlánticas hacia la latitud de Canarias. Esta es una situación sinóptica bastante infrecuente en el archipiélago. El frente asociado a la borrasca de unos $1000 \mathrm{hPa}$ afectó el día 25 a todas las islas, trayendo un flujo del suroeste con vientos muy fuertes de esta dirección y precipitaciones asociadas. Este tipo de situaciones son bastante sensibles y complejas de predecir, ya que pequeños cambios de los modelos durante los días previos respecto a la situación o intensidad de la baja generarían cambios importantes en la emisión de avisos y predicciones asociadas. Es de remarcar además, que al ser una dirección de viento rara en las islas, los daños ocasionados fueron mayores aún, con importantes afecciones al tráfico aéreo y a las comunicaciones terrestres.

Finalmente se decide emitir avisos amarillos de rachas máximas, RM (umbral de $70 \mathrm{~km} / \mathrm{h}$ ), y de precipitación acumulada en 1 hora, P1 (umbral de $15 \mathrm{~mm} / \mathrm{h}$ ), en las islas orientales (Lanzarote y Fuerteventura). En el resto de islas se aumentan a naranja (RM con umbral de $90 \mathrm{~km} / \mathrm{h}$ y P1 con umbral de $30 \mathrm{~mm} / \mathrm{h}$ ) y se añaden avisos naranjas de precipitación acumulada en 12 horas, P2 (umbrales de 40 mm en Gran Canaria y 60 mm en el resto de islas occidentales).

a)

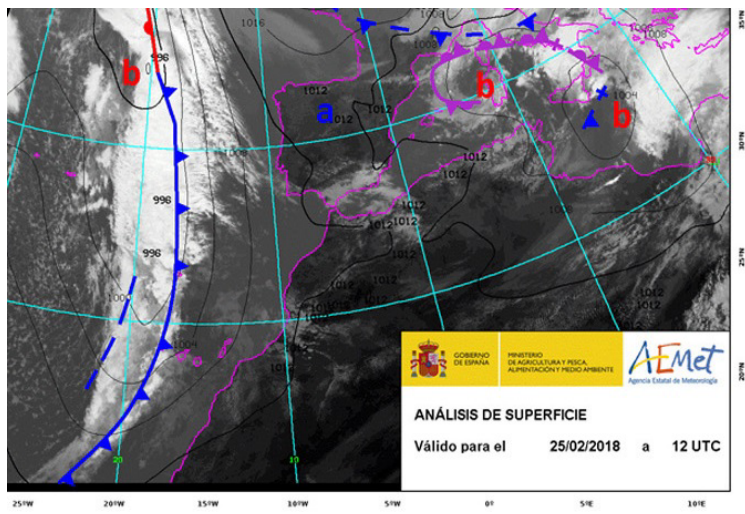

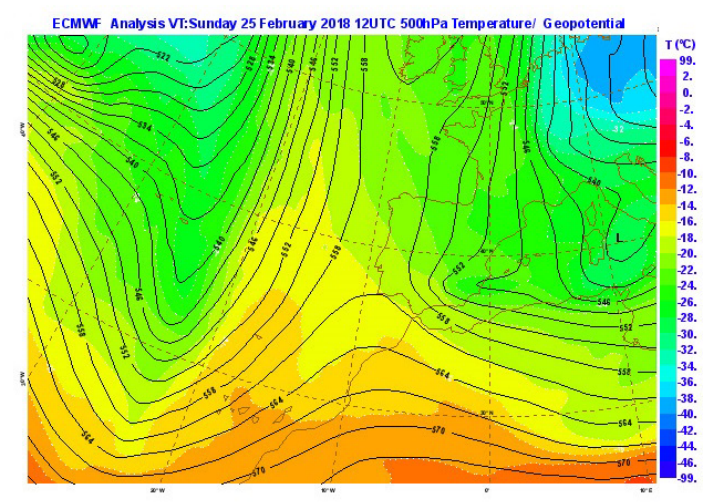

b)

Figura 3. a) Mapa de análisis de presión en superficie con imagen de satélite para las 12 Z del 25-02-2018 y b) temperatura y geopotencial en $500 \mathrm{hPa}$ (Fuente: AEMET).

En la figura 4 se muestran las salidas de rachas máximas y precipitaciones acumuladas en $24 \mathrm{~h}$ de los dos ciclos de Harmonie en este día y se comparan con los datos recopilados por las estaciones automáticas.

Con respecto a las precipitaciones, se puede observar cómo Har38 las predice de forma más generalizada, extendiéndolas hasta Fuerteventura e intensificándolas en las islas occidentales y Tenerife $(90 \mathrm{~mm} / 24 \mathrm{~h})$. En el caso de Har40, las lluvias son ligeramente menos intensas, excepto en Gran Canaria, donde se acercan a los $50 \mathrm{~mm} / 24 \mathrm{~h}$. Las observaciones se aproximaron más a lo previsto por Har40, siendo las precipitaciones reales de $65-75 \mathrm{~mm} / 24 \mathrm{~h}$ en las islas occidentales y llegando hasta los $64 \mathrm{~mm} / 24 \mathrm{~h}$ en la isla de Gran Canaria. 
a)

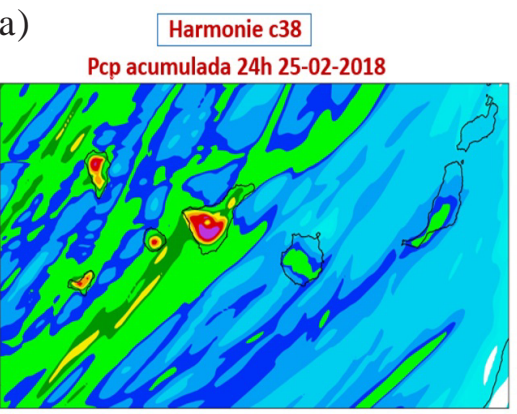

d)

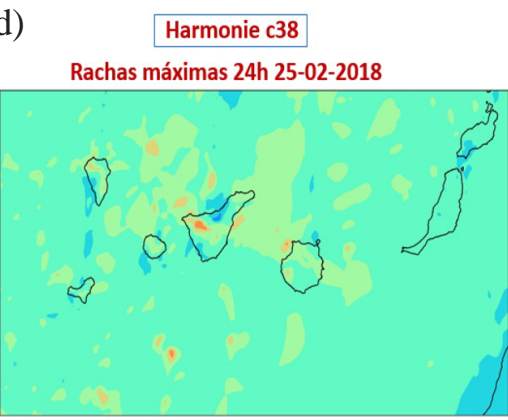

b)

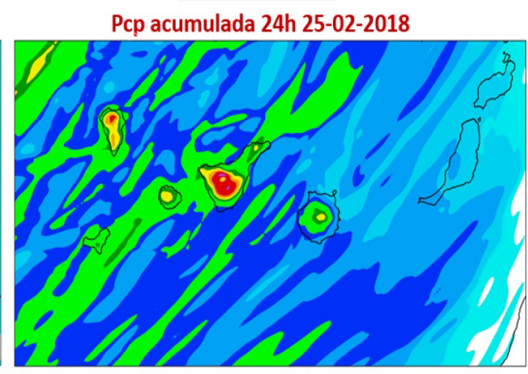

e)

e)

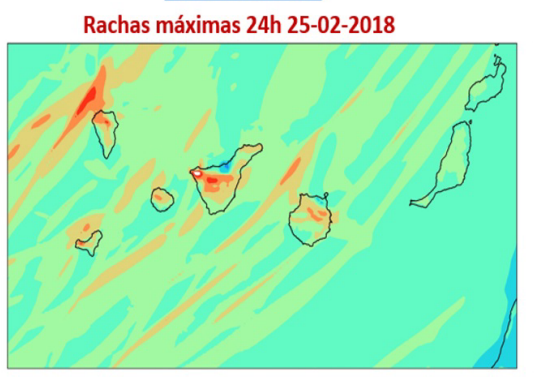

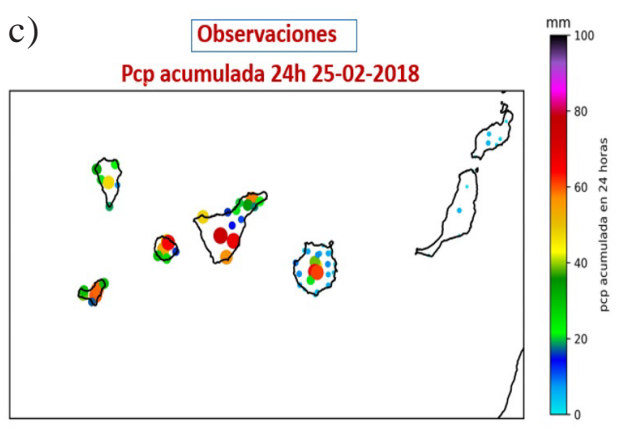

f)

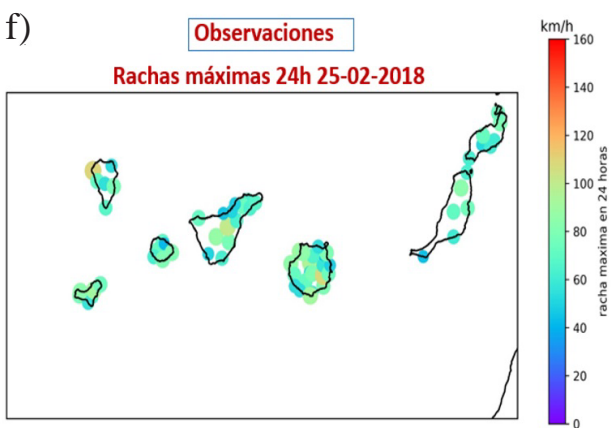

Figura 4. a) Precipitación acumulada en 24 h (mm) pronosticada por Har38, b) Har40 y c) observada y d) rachas máximas en 24 h (km/h) pronosticadas por Har38, e) Har40 y f) observadas, para el día 25-02-2018.

Las rachas máximas de viento en estas 24 h, son mayores en Har40, llegando a $130 \mathrm{~km} / \mathrm{h}$ (umbral de aviso rojo), cuando en Har38 las rachas están, en general, entre $80 / 90 \mathrm{~km} / \mathrm{h}$, con picos locales de $110 \mathrm{~km} / \mathrm{h}$. Finalmente, las rachas observadas fueron, como máximo, de $100-110 \mathrm{~km} / \mathrm{h}$, cumpliéndose de esta forma los avisos naranjas emitidos. En este caso se ilustra la discrepancia entre un modelo y otro respecto a la toma de decisiones importantes como puede ser la emisión de un aviso rojo.

\subsection{Día 07/02/2018. Viento del NW/N (aviso naranja)}

Durante este episodio, las islas se ven afectadas por una típica irrupción de aire polar marítimo, asociada a vaguadas que cruzan el archipiélago, situación relativamente frecuente en Canarias. Los efectos principales de este tipo de situaciones son la bajada de temperaturas, más significativas en niveles altos que a nivel del mar, por lo que suelen romper o debilitar la inversión térmica característica del alisio. Las precipitaciones pueden llegar a ser muy importantes en función de la inestabilidad, contenido en vapor de agua de la masa de aire, ambiente previo, etc., estando muy favorecido por el factor relieve (FonT TULLOT, 1956).

En este episodio en concreto, la situación sinóptica está marcada por la presencia de un potente anticiclón en superficie de unos $1048 \mathrm{hPa}$ al norte de Azores, que aporta un flujo de componente norte, fresco y húmedo al entorno de las islas. También destaca en superficie la presencia de una baja de unos $1006 \mathrm{hPa}$, situada entre la mitad oriental de la península ibérica y el norte de África, que produce un marcado gradiente de presiones, acelerando el flujo del norte sobre las islas. Así, los vientos asociados son muy fuertes, del NW girando a $\mathrm{N}$.

En el nivel de $500 \mathrm{hPa}$, Canarias inicialmente queda aguas abajo de la dorsal en la típica estructura en omega de la intensa circulación del oeste. Durante los días 6 y 7, destaca la profundización de la vaguada con el descolgamiento del núcleo frío asociado a la baja peninsular y el acercamiento máximo hasta nuestra área de interés. El frente asociado llega en frontólisis y debilitado al archipiélago.

Para los días 7 y 8, se emitieron avisos naranjas de RM $(90 \mathrm{~km} / \mathrm{h})$ en zonas altas de La Palma, Gran Canaria y Tenerife, donde también hubo aviso amarillo por nevadas, NV ( $2 \mathrm{~cm}$ por debajo de $2000 \mathrm{~m})$. En el resto de zonas se emitieron avisos de RM amarillos $(70 \mathrm{~km} / \mathrm{h}$ ) y avisos de tormentas, TO, en Fuerteventura y Lanzarote. 
a)

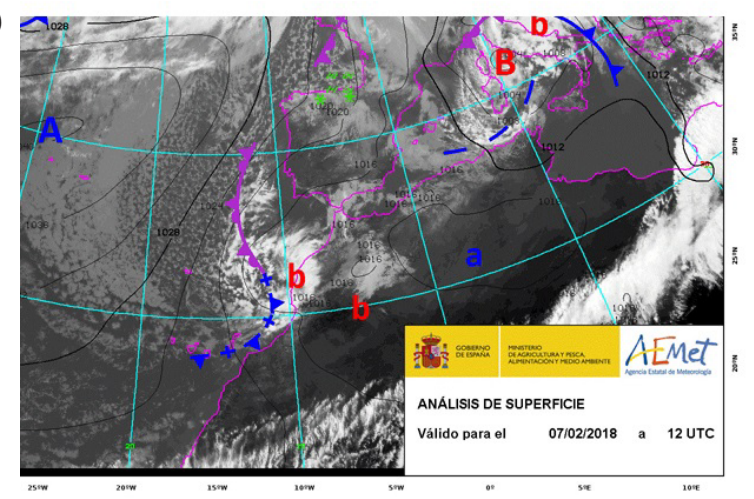

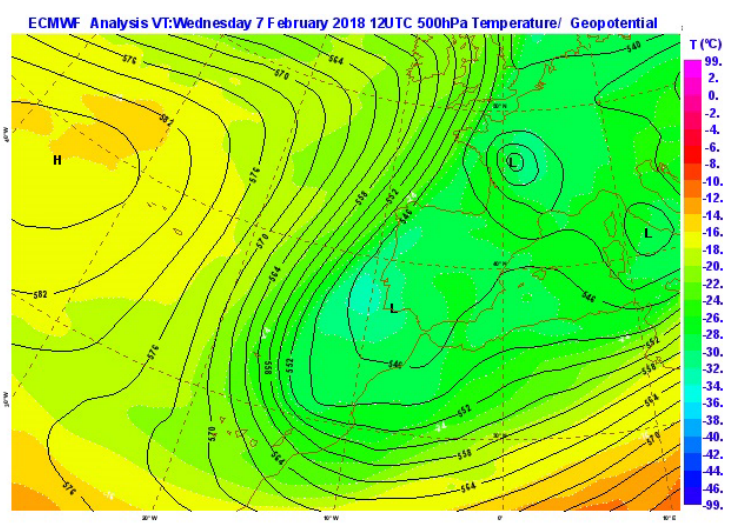

Figura 5. a) Mapa de análisis de presión en superficie con imagen de satélite de las 12 Z del 07-02-2018 y b) temperatura y geopotencial en $500 \mathrm{hPa}$. (Fuente: AEMET).

En la figura 6 se muestra de nuevo la comparación de precipitación y rachas máximas en 24 horas de ambos ciclos de Harmonie-Arome y los datos de observación de las estaciones automáticas de la red de AEMET.

Las precipitaciones registradas dejaron unos 15-20 mm en el norte de Tenerife, que se ven mejor reflejados por Har40, mientras que Har38 presenta valores bastante superiores $(50 \mathrm{~mm})$. En el caso de Gran Canaria, donde se recogieron 30-50 mm, ambos ciclos subestimaron la precipitación con diferencias de 10-15 mm.

En el caso de las rachas máximas de viento, en la isla de Gran Canaria hubo rachas de hasta $94 \mathrm{~km} / \mathrm{h}$ en cumbres, que fueron bien reflejadas por Har40. Sin embargo, las rachas observadas de $70-80 \mathrm{~km} / \mathrm{h}$ en las islas orientales, Har40 las pronosticó con valores de 100-110 km/h (umbral de aviso naranja). Aquí Har38 fue más certero. A sotavento de Izaña (Tenerife), Har40 sí que pronosticaba rachas ligeramente más intensas, pero sin alcanzar los $150 \mathrm{~km} / \mathrm{h}$ que fueron observados.

a)

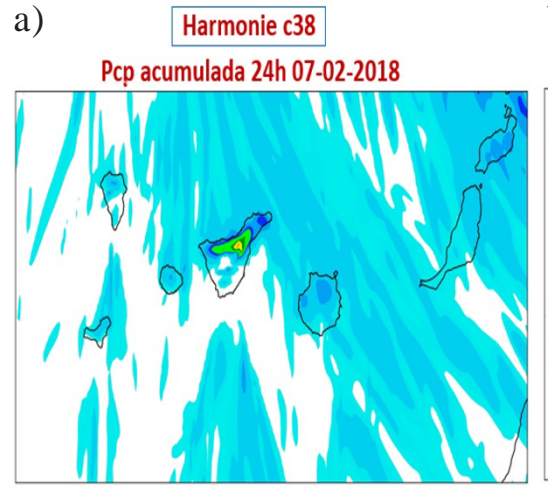

d)

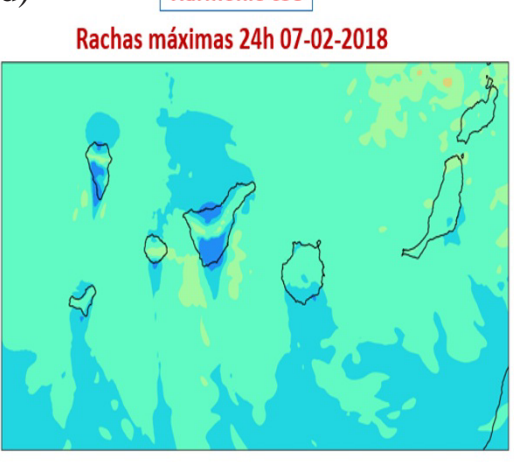

b)

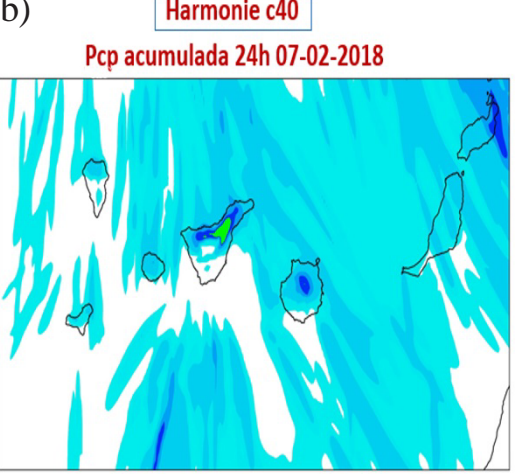

e)

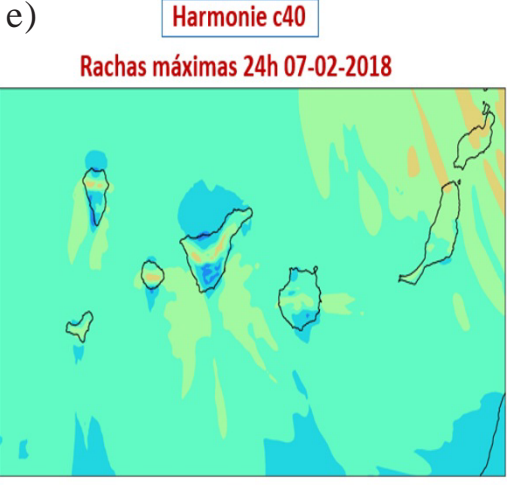

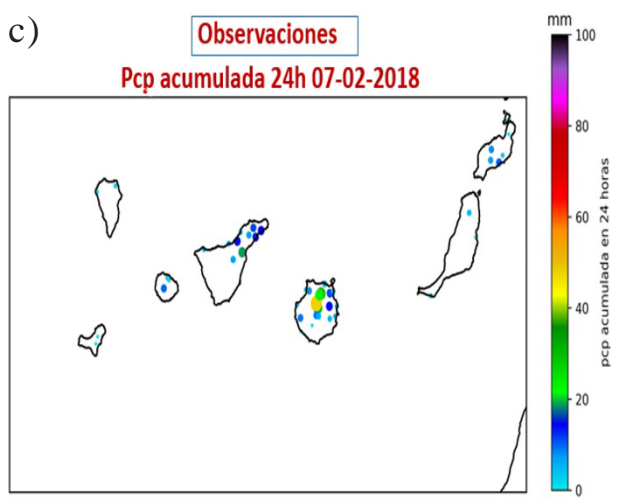

f)

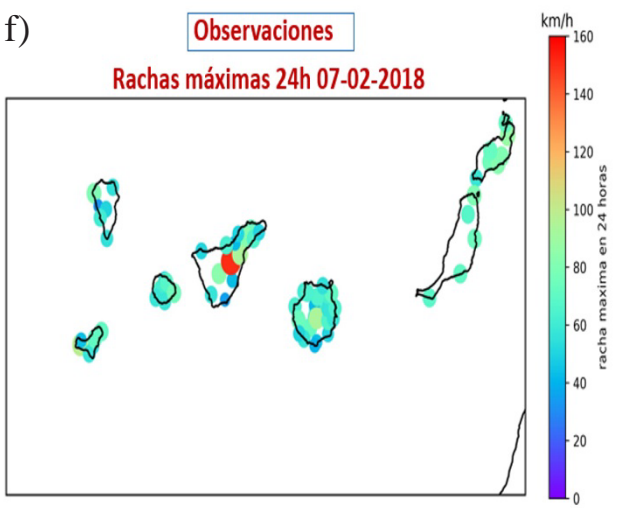

Figura 6. a) Precipitación acumulada en 24 h (mm) pronosticada por Har38, b) Har40 y c) observada y d) rachas máximas en $24 \mathrm{~h}(\mathrm{~km} / \mathrm{h})$ pronosticadas por Har38, e) Har40 y f) observadas, para el día 07-02-2018. 


\subsection{Día 27/01/2018. Viento del NE o alisio intenso (aviso amarillo)}

Entendemos por alisio la circulación en torno al anticiclón caliente subtropical. El archipiélago canario se encuentra en enero en el límite septentrional normal de la región de los alisios del NE. En el transcurso del año, este límite se va desplazando hacia el norte, de forma que en junio ocupa su posición más septentrional. Puesto que en verano sopla con una frecuencia superior al $90 \%$ y en invierno su frecuencia es algo mayor al resto de tipos de tiempo, podríamos decir que el alisio es el tipo de tiempo normal en el archipiélago (FonT Tullot, 1956).

El 27 de enero de 2018 se caracterizó por la presencia de un potente anticiclón de las Azores afectando a superficie con vientos fuertes del NE. En estas situaciones, la inversión térmica característica del alisio, que separa una capa húmeda inferior de una capa superior seca, hace que la inestabilidad quede limitada a la primera de ellas, por lo que la convección es débil, con nubosidad de tipo cumuliforme. Así, las precipitaciones suelen ser débiles y quedar localizadas en el norte/nordeste de las islas. Al ser esta la situación de tiempo normal en Canarias, las infraestructuras de las islas y el tráfico aéreo están mejor preparados cuando el viento es del NE y los impactos son menores aunque el nivel de aviso sea el mismo que en otros eventos.

Los avisos emitidos ese día fueron amarillos para todo el archipiélago, por rachas máximas mayores de 70 km/h. La comparación entre los ciclos de Harmonie-Arome y las observaciones de las estaciones automáticas de rachas máximas y precipitación acumulada en 24 horas se muestra en la figura 8.

a)

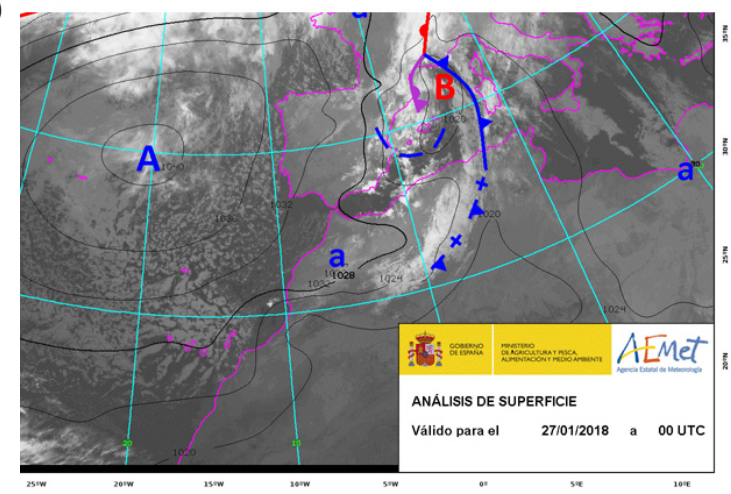

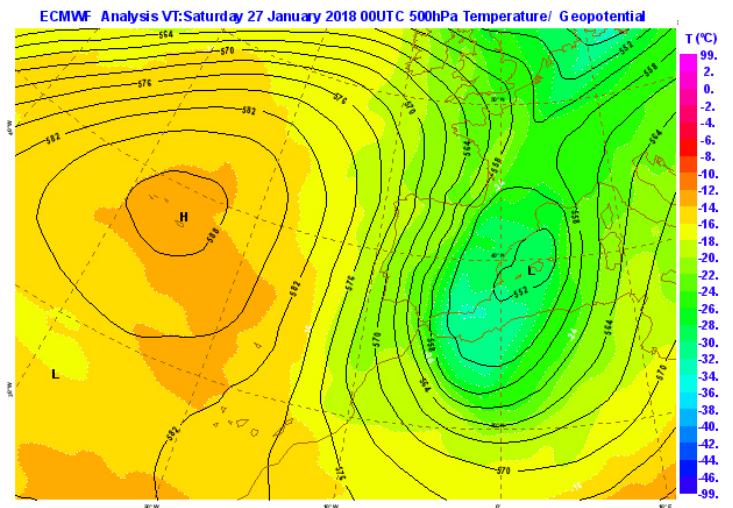

b)

Figura 7. a) Mapa de análisis de presión en superficie con imagen de satélite de las 00 Z del 27-01-2018 y b) temperatura y geopotencial en $500 \mathrm{hPa}$. (Fuente: AEMET).

a)

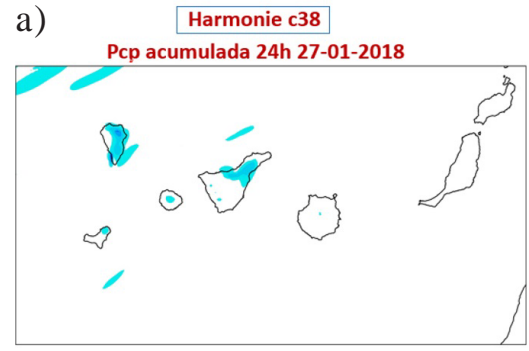

d)

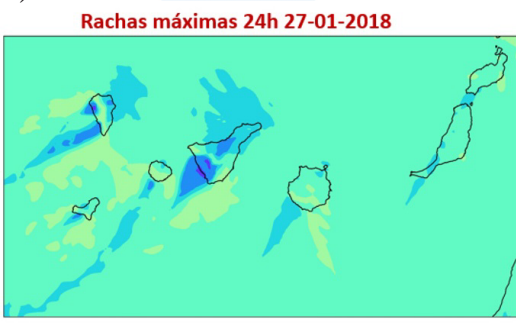

b) $\begin{gathered}\text { Harmonie } \text { } 40 \\ \text { Pcp acumulada 24h 27-01-2018 }\end{gathered}$

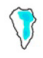

\section{$\sqrt{7}$}

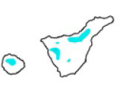

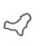

e)

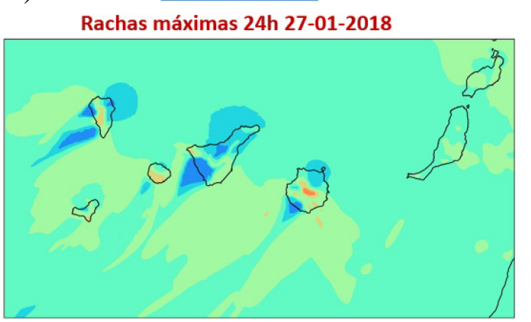

c)

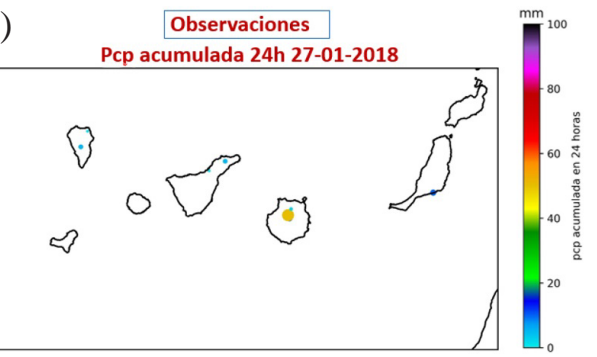

f)

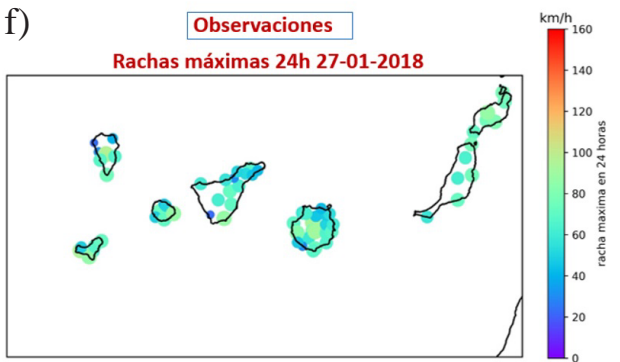

Figura 8. a) Precipitación acumulada en 24 h (mm) pronosticada por Har38, b) Har40 y c) observada y d) rachas máximas en 24 h (km/h) pronosticadas por Har38, e) Har40 y f) observadas, para el día 27-01-2018. 
A pesar de que la precipitación en este episodio no es significativa en cuanto a avisos, se puede observar cómo Har40, aunque subestima el valor de los 50 mm recogidos en Gran Canaria, sí que sitúa la zona de precipitación de forma más precisa que Har38.

Las rachas máximas pronosticadas por Har38 fueron aproximadamente de unos $80-90 \mathrm{~km} / \mathrm{h}$, mientras que Har40 las intensificaba, especialmente en vertientes a sotavento, con valores hasta $100-110 \mathrm{~km} / \mathrm{h}$, llegando al umbral de aviso naranja. Las rachas máximas observadas fueron de $90-100 \mathrm{~km} / \mathrm{h}$, justo a medio camino entre los dos pronósticos. Se decidió emitir un aviso amarillo alto, a pesar de que quizá se podría haber emitido un aviso amarillo, ya que al ser la dirección del viento la normal en Canarias (alisio) los impactos son mucho menores debido a que las infraestructuras están mejor preparadas.

\section{CONCLUSIONES}

Los estudios regionalizados de las capacidades de diferentes modelos numéricos en situaciones de FMA permiten mitigar la controversia a la que deben enfrentarse los predictores operativos a la hora de tomar decisiones en cuanto a la emisión de avisos. Se hace, de hecho, importante la labor interpretativa del predictor a la hora de comparar entre los dos modelos, estudiar otros campos y tomar decisiones. En el caso particular de este estudio, se desprenden las siguientes impresiones:

- Para la intensidad del viento se ve cómo Har40 pronostica rachas de viento mucho más intensas, pero con mejor localización. Para el viento medio ocurre lo mismo pero siendo el pronóstico menos exagerado que para las rachas. En el caso de Har38, en ciertas ocasiones se produce una subestimación de la intensidad.

- Respecto a la precipitación, el estudio visual se muestra más complicado por falta de datos comparables y por la mayor similitud entre los modelos, pero la percepción es de una excesiva convección en el caso de Har38.

Finalmente, esto plantea la controversia de cuál es la predicción más óptima, una predicción más alarmista o sobrepredicción con más casos de falsas alarmas, o una más conservadora que puede dar lugar a un mayor número de fallos. La necesidad de unificar criterios se hace patente aquí. Otro aspecto a tener en cuenta es el futuro probabilista de los modelos de predicción, difícilmente aplicable en aeropuertos. El tener que comparar varios modelos crea incertidumbre en predicciones que se requieren fundamentalmente deterministas, como en el caso de la aeronáutica. Además, crea al predictor una sensación de sobresaturación de información, que a nivel operativo se hace fatigante. El predictor finalmente adquiere un criterio más o menos subjetivo acerca de la fiabilidad de cada modelo. Por lo que es bueno establecer las diferencias y el modo de comportamiento de los diferentes modelos. Así, se requiere un mayor estudio, cooperación y comunicación predictor-desarrollador en cuanto a la verificación y desarrollo futuro de los modelos utilizados.

\section{AGRADECIMIENTOS}

A los compañeros del Grupo de Predicción y Vigilancia de Canarias, que han contribuido con su trabajo diario al desarrollo de este estudio, así como al resto de personal de la Delegación Territorial de Canarias. Al grupo del Área de Técnicas y Aplicaciones de Predicción (ATAP), por motivar este estudio en el marco del curso PAF 2018 «Nuevos desarrollos operativos en el ATAP(Canarias)».

\section{REFERENCIAS}

Bengtsson, L., Andrae, U., Aspelien, T., Batrak, Y., Calvo, J., de Rooy, W., Gleeson, E., Hansen-Sass, B., Homleid, M., Hortal, M., Ivarsson, K., Lenderink, G., Niemelä, S., Pagh Nielsen, K., Onvlee, J., 
Rontu, L., Samuelsson, P., Santos Muñoz, D., Subias, A., Tijm, S., Toll, V., Yang, X. y Ødegaard KøLTzow, M., 2017. The HARMONIE-AROME model configuration in the ALADIN-HIRLAM NWP system. Mon. Wea. Rev. doi: https://doi.org/10.1175/MWR-D-16-0417.1.

Font Tullot, I., 1956. El tiempo atmosférico en las Islas Canarias.

ALADIN-HIRLAM, 2012. Release Notes on Harmonie 38h1.2: https://www.hirlam.org/trac/wiki/ ReleaseNotes/harmonie-38h1.2.

ALADIN-HIRLAM, 2014. Release Notes on Harmonie 40h1: https://www.hirlam.org/trac/wiki/ ReleaseNotes/harmonie-40h1.1.

WWRP/WGNE Joint Working Group on Forecast Verification Research, 2017. 7th International Verification Methods Workshop: http://www.cawcr.gov.au/projects/verification/. 
